\title{
Book Review - Bridging the Knowledge Divide: Educational Technology for Development
}

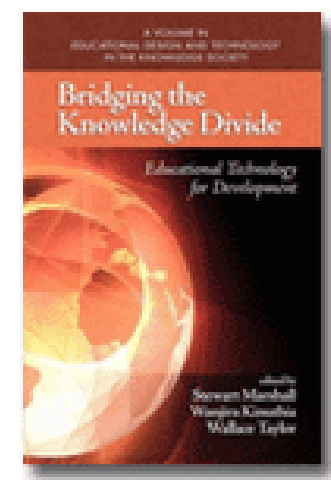

Reviewer: Aminudin Zuhairi, Universitas Terbuka, Indonesia

Addressing the challenge of bridging the knowledge divide through the use of educational technology, this book is organized around four main themes: empowerment, knowledge, flexible delivery, and teacher preparation. Contributors to the discussion of these themes raise strong alarms about the potential pitfalls of the shift from a digital divide to a knowledge divide, calling for planned action. Drawing on their experience with education technology and distance education policy, theory, practice, and implementation in different societal and cultural contexts, in both developed and developing countries, some 40 contributors present roadmaps and strategies for planning and implementing educational technology that can bridge the knowledge divide.

Opinions about the knowledge divide differ widely, with pessimists perceiving the knowledge divide growing wider. On the other hand, optimists, such as the book's editors and contributors, share a common view that the knowledge divide can be bridged through establishment of technology literacy and effective use of technology. Indeed, the main aim of the book is to show "how educational technology can utilize ICT to transform education and to assist developing communities in closing the knowledge divide" (p. xxviii).

The book offers theoretical and philosophical insights, presents benchmarks and best practices, and analyses research results that inform readers and decision makers about actions that can address the issues involved in bridging the knowledge divide. Case studies provide both broad overviews and specific instances from which readers can learn what approaches and actions may be taken to bridge the knowledge gap. Chapter authors present evidence from developed and developing country contexts to support the conclusion that governments and societies can bridge the knowledge media gap through effective uses of 
technology for development. Arguments are well supported with statistical data, facts, and research findings from a wide variety of social and cultural contexts.

Following the book's introduction, four sections, comprising 23 chapters, address issues that pertain to flexible education for empowerment, managing and communicating knowledge, flexible delivery in higher education, and preparation of teachers using flexible approaches. Despite conflicting views as to whether advances in knowledge benefit different societies and cultures fairly, there is growing acceptance of the perception that all societies will eventually become knowledge societies. Chapters on flexible education for empowerment cover issues related to community development, community economic development, gender, empowerment of women, enablers and constraints of ICTs in higher education, and civil service training. Chapters on managing and communicating knowledge address issues related to knowledge management strategies; the effectiveness of mobile technology; and the impact of video conferencing, open resources, open source software, and copyright issues. Chapters on flexible delivery in higher education discuss national development, policy considerations, blended online and face-to-face learning, cognitive-based learning, shifts from distance to e-learning, cross-regional delivery, and postgraduate medical education. Chapters on teacher preparation using flexible approaches address issues related to preservice teacher preparation using e-learning, costs of distance teacher training, computer technology for e-learners, use of educational technology, and digital literacy of primary teachers.

An abundance of insights, examples, and good practices, one of the book's major strengths, can inspire educational theorists, researchers, policy makers, and practitioners as they construct and implement new approaches to confront the challenge in their own contexts. The book presents concise and comprehensive analyses of actions addressing knowledge for development worldwide. It will not only serve as good reference material but also enrich the literature of distance education theory, research, and practice.

There is no doubt that we can learn a great deal from this book. In his Foreword, Sir John Daniel of the Commonwealth of Learning states that the book "will help to bridge the knowledge divide, an essential task in bringing closer together the extraordinary extremes of this world in which we live" (p. xi). Confronted with the paradox of the knowledge divide, educators must find ways of addressing this challenge through integration of ICT in "the development, delivery, and content of education" (p. xxvii). Reading this book will trigger awareness of barriers to equitable distribution of traditional forms of educational services. "Developing countries should therefore seek to adopt as far as possible the tools for effective delivery of these alternative forms of education" (p. 17). New technological development helps societies to advance equitably. Although "open source communities do not equate to ideal societies” (p. 197), there seems to be growing acknowledgement that open source can promote equitable access to technology.

Because of its systematic and comprehensive approaches to the challenges of bridging the knowledge divide, this book is not to be missed. It will be of great value to readers involved in exploring new theories and approaches and implementing new strategies and effective 
practices in open and distance learning. Readers will gain better and deeper understanding of the issues related to the knowledge divide and insights into new approaches and strategies. The book can thus point us toward the necessary steps in our common efforts to bridge the knowledge divide and foster human development.

\section{Athabasca University $\mathbf{A}$}

(c) 